\section{Longitudinal study of a cohort of people with diabetes screened by the Exeter Diabetic Retinopathy Screening Programme}

${ }^{1}$ West of England Eye Unit Royal Devon \& Exeter Hospital

Exeter, Devon, UK

2Department of Diabetes and Vascular Medicine School of Postgraduate Medicine and Health Science University of Exeter Devon, UK

\section{Correspondence:}

R Ling

West of England Eye Unit Royal Devon \& Exeter

Hospital

Barrack Road

Exeter EX2 5DW, UK

Tel: 01392406002

Fax: 01392406022

E-mail: roland@

linghl.fsnet.co.uk

\begin{abstract}
Purpose To evaluate the long-term outcome of a cohort of people with diabetes screened by the Exeter Diabetic Retinopathy Screening Programme.

Method The cohort comprised of diabetic patients from nine market town General Practices around Exeter who first attended the diabetic retinopathy screening programme in 1992. Screening was carried out by a combination of dilated fundoscopy by a screening technician and a single $45^{\circ}$ Polaroid photograph of each eye. The prevalence of diabetic retinopathy at the baseline, the incidence of sight threatening diabetic retinopathy (STDR) in the next two rounds of screenings, and the long-term visual outcome are described.
\end{abstract}

Results The cohort consisted of 775 patients (104 Type I, 517 Type II non-insulinrequiring and 154 Type II insulin-requiring). The mean age was 72.1 years $(15-100)$, with mean disease duration of 13.0 years (1-79). Baseline prevalence was as follows: background retinopathy: $21.4 \%$, proliferative: $2.8 \%$, clinically significant macular oedema: $6.1 \%$. The incidence of proliferative disease was $2.20 \%$ and $2.25 \%$ respectively in the next two rounds of screening $\left(P>0.05, \chi^{2}\right.$ test). The incidence of clinically significant macular oedema was also constant over next two rounds of screening, at $4.79 \%$ and $5.18 \%$ respectively ( $P>0.05, \chi^{2}$ test). Ninety-five (12.3\%) patients were deceased and a further $179(23 \%)$ patients were lost to follow-up by the third round. Only two patients with newly diagnosed STDR suffered deterioration of visual acuity to $6 / 60$ or worse as a result of diabetic retinopathy.
Conclusion The incidence of STDR remained constant throughout the period of screening, reflecting the chronic progressive nature of the disease. Significant numbers of patients were lost to follow-up. Screening seemed effective in preserving vision in those patients with newly diagnosed STDR. Eye (2002) 16, 140-145. DOI: 10.1038/ sj/EYE/6700081

Keywords: diabetic retinopathy; screening; prevalence; incidence; sight threatening diabetic retinopathy; visual acuity

\section{Introduction}

The impact of visual impairment secondary to diabetic retinopathy on the working age population in the UK is well recognised. ${ }^{1}$ The benefit of timely intervention in the form of laser treatment has also been convincingly demonstrated..$^{2,3}$ It is not surprising therefore that diabetic retinopathy screening programmes are already in place across a number of regions in the UK. ${ }^{4}$

As we approach the threshold of implementing a national diabetic retinopathy screening programme within the $\mathrm{UK}^{5}$ questions concerning the impact of screening remain unanswered. There is as yet no published evidence to suggest that screening can result in a reduction in registrable blindness in a diabetic population. In addition, visual impairment due to diabetic retinopathy does not appear to be the major cause of severe visual loss in people with diabetes, especially those with Type II diabetes. ${ }^{6}$

If blindness is too crude an outcome 
measure to usefully assess the impact of screening, alternative outcome indicators will have to be considered. Our aim is to examine some of these alternatives in this paper. We begin by describing the baseline prevalence of diabetic retinopathy in a cohort of people with diabetes at the inception of our screening programme in 1992. The impact of screening on the incidence of sight threatening diabetic retinopathy (STDR) in this cohort, and the visual acuity (VA) of new patients identified with STDR, is studied over two subsequent screening rounds.

\section{Methods}

This is a retrospective, longitudinal study of a cohort of people with diabetes screened by the Exeter Diabetic Retinopathy Screening Programme between 1992 and 1998. Data were obtained from the Screening Programme database. Data entry took place within 2-3 weeks after each screening episode. The same screening technician (DT) was responsible for data entry and the maintenance of the database throughout the study period.

\section{The study cohort}

Patients with diabetes from nine market town general practices around Exeter were selected as the cohort for this study. We had deliberately chosen a population from a 'rural' community. We assumed that this 'rural population' would be geographically more stable compared with a 'city population', and therefore more suitable for this longitudinal study. The cohort was amongst the first patients screened by the Exeter Diabetic Retinopathy Screening Programme when it was introduced in 1992. Prior to this, there was no organised diabetic retinopathy screening in the community. Biannual screenings were performed thereafter and data were available for two subsequent rounds of screening.

\section{The screening programme}

Details of our screening programme had previously been reported. ${ }^{7}$ Patients with diabetes were identified from the diabetic registry kept by each individual general practice. All patients were then invited to attend screening clinics at their practices, including those who were already under the supervision of hospital ophthalmologists.

The same retinal screener (DT) performed screening in all the patients throughout the study period. Data on patient demography and diabetic management category were obtained. Snellen visual acuity was recorded prior to pupil dilation with $1 \%$ Tropicamide. A single $45^{\circ}$ retinal photograph centred midway between the macula and the optic disc was taken with a mobile Canon Non-Mydriatic polaroid camera, followed by fundus examination by the screener using the direct and indirect ophthalmoscope. An assessment was also made on the degree of cataract and any other non-diabetic ophthalmological findings. The polaroid photographs were subsequently scrutinised by an ophthalmologist with a main interest in diabetic retinopathy $(\mathrm{JJ})$, before the final report and management recommendations were made to the general practitioners.

\section{Retinopathy grading}

The Exeter Diabetic Retinopathy Screening Programme grading and the ETDRS equivalent are summarised in Table 1. Grading for each patient was given according to the worst eye. Patients in Grade 0 and 1 were screened biannually. Patients in Grade 2 were recommended to have their diabetic control reviewed by their general practitioners, and might be recalled for annual retinopathy screening. In addition, a subset of patients in Grade 2 with severe background (preproliferative) changes was referred to the hospital ophthalmic service. By definition, retinopathy in Grades 3 and 4 is sight threatening (STDR). Patients in Grade 3 and 4 were recommended for urgent referral to the hospital ophthalmologists. All referrals were made to one ophthalmic unit (The West of England Eye Unit, Exeter). General practitioners were also alerted to patients with significant non-diabetic ophthalmological findings, such as cataract, suspect optic discs and retinal vein occlusions, for appropriate ophthalmological referrals.

Table 1 Grading of diabetic retinopathy in the Exeter Diabetic Retinopathy Screening Programme

\begin{tabular}{|c|c|c|}
\hline $\begin{array}{l}\text { Exeter } \\
\text { grade }\end{array}$ & $\begin{array}{l}\text { ETDRS } \\
\text { level }\end{array}$ & Summary \\
\hline 0 & 10 & No retinopathy \\
\hline 1 & $20-30$ & Background retinopathy \\
\hline 2 & $40-50$ & $\begin{array}{l}\text { Moderate to severe non-proliferat- } \\
\text { ive retinopathy }\end{array}$ \\
\hline 3 & $60-70$ & $\begin{array}{l}\text { Proliferative retinopathy, DRS high } \\
\text { risk characteristics }\end{array}$ \\
\hline 4 & $\begin{array}{l}\text { ETDRS } \\
\text { CSMO }\end{array}$ & $\begin{array}{l}>\text { five microaneurysms or haemor- } \\
\text { rhage within one disc diameter from } \\
\text { fovea, and ETDRS definition of clini- } \\
\text { cally significant maculopathy }\end{array}$ \\
\hline 5 & 90 & $\begin{array}{l}\text { Ungradable for any reason, plus } \\
\text { Central or branch vein occlusions }\end{array}$ \\
\hline
\end{tabular}

DRS, Diabetic Retinopathy Study; ETDRS, Early Treatment Diabetic Retinopathy Study; CSMO, clinically significant macular oedema. 


\section{Statistical analysis}

The prevalence of retinopathy, the incidence of proliferative retinopathy (nPDR) and the incidence of clinically significant maculopathy (nCSM) were compared using the $\chi^{2}$ test. A $P$-value of $<0.05$ was used as the level of significance.

\section{Results}

\section{Screening round 1 (July 1992-December 1993)-the baseline}

A total of 912 diabetic patients were identified from the diabetic register of the nine practices and invited for screening. Seven hundred and seventy-five (85\%) attended Round 1 and constituted the study cohort. The mean age of the cohort was $72.1( \pm 14.5)$ years, with a range of 15-99 years. There were 420 (54.2\%) males and 355 (45.8\%) females. Diabetic management was categorised as follows: Type I 104 (13.4\%), Type II non-Insulin requiring (NIR) 517 (66.7\%), Type II Insulin-requiring (IR) 154 (19.9\%). The mean duration of diabetes at Round 1 was $13.0( \pm 9.7)$ years (range 1-79).

The prevalence of diabetic retinopathy at Round 1 is presented in Table 2. Three hundred and ten $(40.0 \%)$ of the cohort had cataracts of various degrees. Other incidental ophthalmological findings included nine (1.2\%) glaucomatous optic discs, 19 (2.5\%) combined cataracts and glaucomatous optic discs, six $(0.8 \%)$ retinal vein occlusions, and $14(1.8 \%)$ significant macular drusen/disciform macular degeneration.

Baseline visual acuity (VA) was 6/9 or better in 602 $(77.7 \%)$ patients, $6 / 12$ to $6 / 24$ in $161(20.8 \%)$ and $6 / 36$ or worse in $12(1.5 \%)$ patients. In the 12 patients with $\mathrm{VA}<6 / 36$, six were due to cataracts, four age-related maculopathy and only two were due to diabetic retinopathy.
Screening round 2 (January 1995-January 1996) $\mathcal{E}$ screening round 3 (March 1997-February 1998)

The cohort was screened on two subsequent rounds of screening; Round 2 at $1.8( \pm 0.24)$ years and Round 3 at $4.3( \pm 0.32)$ years after Round 1 . There was $100 \%$ data retrieval of all the screening episodes from the Screening Programme database.

The prevalence of diabetic retinopathy (including patients with previously known and newly diagnosed retinopathy) over the three consecutive rounds of screenings is detailed in Table 3 . There is a significant increase in background retinopathy $\left(P<0.001, \chi^{2}\right.$ test), but no significant change in the prevalence of PDR and CSM ( $P>0.10, \chi^{2}$ test) over three rounds of screening.

New cases of sight threatening diabetic retinopathy emerged during each round of screenings. The incidence of proliferative retinopathy (nPDR) and clinically significant maculopathy (nCSM) in Rounds 2 and 3 is summarised in Table 4. nPDR and nCSM were not significantly different between Rounds 2 and 3 ( $P$ $>0.05, \chi^{2}$ test).

\section{Visual outcome of new cases of STDR}

Round 1 Of the 47 patients with maculopathy identified during initial screening, $12(26 \%)$ required immediate focal laser photocoagulation following referral to the hospital ophthalmic service. One patient (2\%) had a reduction in VA from $6 / 12$ to $6 / 60$ in the worse eye secondary to maculopathy by Round 3. All 22 patients with proliferative retinopathy in Round 1 retained their baseline VA by Round 3 .

Round 2 There were 28 cases with newly diagnosed maculopathy. Nine (32\%) required immediate laser photocoagulation following ophthalmological referrals. One patient (4\%) suffered a deterioration of VA from $6 / 12$ to $6 / 60$ secondary to diabetic maculopathy by

Table 2 Baseline prevalence of diabetic retinopathy in Round 1

\begin{tabular}{lcccc}
\hline & $\begin{array}{c}\text { Type I (\%) } \\
(n=104)\end{array}$ & $\begin{array}{c}\text { NIR Type II (\%) } \\
(n=517)\end{array}$ & $\begin{array}{c}\text { IR Type II (\%) } \\
(n=154)\end{array}$ & $\begin{array}{c}\text { Total (\%) } \\
(n=775)\end{array}$ \\
\hline $\begin{array}{l}\text { No retinopathy } \\
\text { Grade 0) }\end{array}$ & $53(51.0)$ & $397(76.8)$ & $90(58.4)$ & $540(69.7)$ \\
$\begin{array}{l}\text { Background retinopathy } \\
\text { Grade 1 and 2) }\end{array}$ & $30(28.8)$ & $92(17.8)$ & $44(28.6)$ & $166(21.4)$ \\
$\begin{array}{l}\text { Proliferative retinopathy } \\
\text { Grade 3) }\end{array}$ & $9(8.7)$ & $7(1.3)$ & $6(3.9)$ & $22(2.8)$ \\
$\begin{array}{l}\text { Clinically significant maculopathy } \\
\text { Grade 4) }\end{array}$ & $12(11.5)$ & $21(4.1)$ & $14(9.1)$ & $47(6.1)$ \\
\hline
\end{tabular}

NIR, non-insulin requiring; IR, insulin-requiring. 
Table 3 Prevalence of retinopathy (previously known and newly diagnosed) in the three rounds of screenings

\begin{tabular}{lccc}
\hline & $\begin{array}{c}\text { Round 1 (\%) } \\
(n=775)\end{array}$ & $\begin{array}{c}\text { Round 2 (\%) } \\
(n=601)\end{array}$ & $\begin{array}{c}\text { Round 3 (\%) } \\
(n=501)\end{array}$ \\
\hline No retinopathy (Grade 0) & $540(69.7)$ & $368(61.2)$ & $250(49.9)$ \\
Background retinopathy (Grade 1 and 2) & $166(21.4)$ & $165(27.5)$ & $185(36.9)$ \\
Proliferative retinopathy (Grade 3) & $22(2.8)$ & $24(4.0)$ & $23(4.6)$ \\
Clinically significant maculopathy (Grade 4) & $47(6.1)$ & $44(7.3)$ & $43(8.6)$ \\
\hline
\end{tabular}

Table 4 The incidence of proliferative retinopathy and clinically significant maculopathy in the study cohort during Rounds 2 and 3

\begin{tabular}{lcccccc}
\hline & New PDR & Previously PDR-free & $n P D R(\%)$ & New CSM & Previously CSM-free & $n$ CSM (\%) \\
\hline Round 2 & 13 & 590 & 2.20 & 28 & 585 & 4.79 \\
Round 3 & 11 & 489 & $2.25^{*}$ & 25 & 483 & $5.18^{*}$ \\
\hline
\end{tabular}

${ }^{*} P>0.05, x^{2}$ test.

PDR, proliferative retinopathy; CSM, clinically significant maculopathy; $\mathrm{nPDR}$, incidence of proliferative retinopathy in the study cohort; nCSM, incidence of clinically significant maculopathy in the study cohort.

Round 3. There were 13 patients with newly diagnosed proliferative retinopathy in Round 2. None of them suffered a deterioration in VA, and two $(15 \%)$ had $\geq 2$ line improvement in Snellen VA secondary to treatment of their concurrent maculopathy by Round 3.

\section{Cohort attrition}

The number of patients who were lost to screening in Rounds 2 and 3 are detailed in Figure 1. Of the 117 patients who did not attend (DNA) Round 2, 70 (59.8\%) had no diabetic retinopathy, 29 (24.8\%) had background retinopathy, and 18 patients $(15.4 \%)$ had STDR at Round 1. Twelve of the 18 patients with STDR were under hospital ophthalmic follow-up at the time of Round 2.

A further 36 of the 117 who DNA Round 2 returned

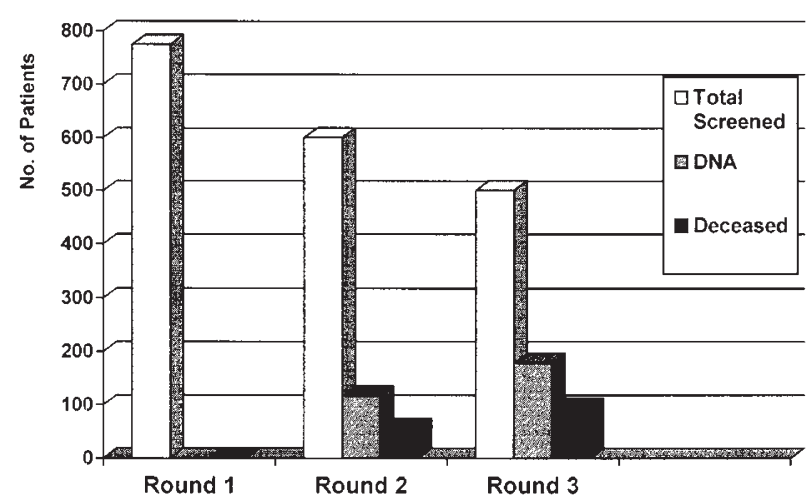

Figure 1 The number of patients who attended screening (total screened), the number who did not attend (DNA), and those who were deceased during the three rounds of retinopathy screening. to the screening programme during Round 3.

Therefore, only 69 of the 117 who DNA Round 2 were truly lost to follow-up by both the screening programme and the hospital ophthalmic service at the end of the study period. This resulted in an adjusted DNA rate of $8.9 \%$ for the original cohort of 775 patients.

\section{Discussion}

The findings of the initial screening round in 1992-93 provided baseline data on the prevalence of diabetic retinopathy in a defined rural diabetic population. The cohort represented approximately one-fifth of all the diabetic patients screened during the first round of the screening programme when it was introduced in 1992. Our baseline prevalence rates are broadly comparable with the rates reported in three other population studies: the Wisconsin Epidemiologic Study of Diabetic Retinopathy (WESDR), ${ }^{8,9}$ the Melton Mowbray study $(\mathrm{MM}){ }^{10,11}$ and the Liverpool Diabetic Eye Study (LDES). ${ }^{12}$ There is one exception: In Type II non-insulin requiring diabetics, which constituted the largest subgroup in all three UK studies (MM 62.4\%, LDES $75.1 \%$, Exeter $66.7 \%$ ), our prevalence rate was significantly lower than MM but more comparable with the LDES (any retinopathy: MM 52\%, LDES 31.3\%, Exeter 23.2\%; PDR: MM 4\%, LDES 1.1\%, Exeter 1.3\%; CSM: MM 10\%, LDES 5.7\%, Exeter 4.1\%).

In contrast to the screening methods of the other population studies, ${ }^{8-12}$ which involved multi-field photography and clinical examination by ophthalmologists, we combine single-field photography with screening technician ophthalmoscopy. ${ }^{7}$ The impact 
of adding screener ophthalmoscopy to the Polaroid imaging in our screening set-up is such that a sensitivity of $95 \%$ and specificity of $97 \%$ can be achieved for the detection of STDR in a direct comparison with 7-fields stereo photography grading by the Hammersmith Grading Centre. ${ }^{13}$

It had previously been suggested that the incidence of STDR in a diabetic population would decrease with successive rounds of screening. Taylor ${ }^{4}$ reported a decrease in both the percentages of people referred to ophthalmologists and the percentages of all screening episodes that result in laser treatment in four UK centres. However, a constant incidence of STDR was found throughout our study. Our findings probably reflect the chronic progressive nature of diabetic retinopathy, with longer disease duration being a significant risk factor for the development of retinopathy in both Type I and Type II diabetics. ${ }^{8-11}$ Our cohort was on average 4.3 years older by Round 3 . There had been a gradual but significant increase in background diabetic retinopathy from Round 1 to Round $3(21.4-36.9 \%)$. It is perhaps not surprising that new cases of STDR emerged at a constant rate throughout the two subsequent rounds of screening.

The natural history of untreated STDR is well known. ${ }^{2,3}$ It is encouraging to find that the majority of patients found to have STDR did not suffer significant deterioration in their vision during the study period. However, without direct comparison with a control, unscreened cohort, it is difficult to draw firm conclusions on our visual findings alone.

A screening programme is only effective if there is consistently high-uptake among the target population. Despite an initial attendance rate of $85 \%$, nonattendance appeared to be a significant problem in the two subsequent screening rounds. The reasons for nonattendance were unclear. The majority of those who did not attend had no retinopathy at the baseline. There is some evidence that these patients were perhaps excessively reassured by their baseline findings, and therefore did not feel compelled to attend the next round of screening 2 years later. ${ }^{14}$ At present, we are not aware of any evidence to suggest that more frequent annual screening can result in a reduction of the non-attendance rate.

\section{Conclusions}

We reported the baseline prevalence of diabetic retinopathy in a defined cohort of rural, mainly elderly diabetic patients, when the Exeter Diabetic Retinopathy Screening Programme was first introduced in 1992. Despite a significant increase in the prevalence of background diabetic retinopathy after two further rounds of screening over the next 4 years, the incidence of sight threatening diabetic retinopathy remained constant. The majority of new patients diagnosed with sight threatening diabetic retinopathy did not suffer a reduction of visual acuity, suggesting that the screening programme was effective in identifying these at-risk individuals for timely interventions.

\section{Acknowledgements}

Proprietary interest, none. Financial support: West of England Eye Unit Trust Fund. Poster presentation at The Royal College of Ophthalmologists Annual Congress 2001.

\section{References}

1 Evans J, Rooney C, Ashwood F, Dattani F, Wormold R. Blindness and partial sight in England and Wales: April 1990-March 1991. Health Trends 1996; 28: 5-12.

2 Diabetic Retinopathy Study Research Group. Photocoagulation treatment of proliferative diabetic retinopathy. Ophthalmology 1981; 88: 538-600.

3 Early Treatment Diabetic Retinopathy Study Research Group. Photocoagulation for diabetic macular edema. Ophthalmology 1986; 93: 938-950.

4 R Taylor. Practical community screening for diabetic retinopathy using the mobile retinal camera: report of a 12 centre study. Diab Med 1996; 13: 946-952.

5 Gillow JT, Muir Gray JA. The National Screening Committee review of diabetic retinopathy screening. Eye 2001; 15: 1-2.

6 Rhatigan MC, Leese GP, Ellis J, Ellingford A, Morris AD, Newton RW, Roxburgh STD. Blindness in patients with diabetes who have been screened for eye disease. Eye 1999; 3: 166-169.

7 J Jacob, J Stead, J Sykes, D Taylor, JE Tooke. A report on the use of technician ophthalmoscopy combined with the use of the Canon non-mydriatic camera in screening for diabetic retinopathy in the community. Diab Med 1995; 12 419-425.

8 Klein R, Klein BEK, Moss SE, Davis MD, DeMets DL. The Wisconsin Epidemiologic Study of Diabetic Retinopathy. II. Prevalence and risk of diabetic retinopathy when age at diagnosis is less than 30 years. Arch Ophthalmol 1984; 102: $520-526$.

9 Klein R, Klein BEK, Moss SE, Davis MD, DeMets DL. The Wisconsin Epidemiologic Study of Diabetic Retinopathy. II. Prevalence and risk of diabetic retinopathy when age at diagnosis is 30 or more years. Arch Ophthalmol 1984; 102: $527-532$.

10 McLeod BK, Thompson JR, Rosenthal AR. The prevalence of retinopathy in the insulin-requiring diabetic patients of an English country town. Eye 1988; 2: 424-430.

11 Sparrow JM, McLeod BK, Smith TDW, Birch MK, Rosenthal AR. The prevalence of diabetic retinopathy and maculopathy and their risk factors in the non-insulintreated diabetic patients of an English town. Eye 1993; 7: 158-163.

12 Broadbent DM, Scott JA, Vora JP, Harding SP. Prevalence 
of diabetic eye disease in an inner city population: the Liverpool Diabetic Eye Study. Eye 1999; 13: 160-165.

13 Taylor DJ, Fisher J, Jacob J, Tooke JE. The use of digital cameras in a mobile retinal screening environment. Diabetic Medicine 1999; 16: 1-7.
14 Eiser JR, Eiser C, Riazi A, Taylor DJ, Hammersley S, Tooke JE. Screening for diabetic retinopathy is well received by patients and may improve self-management intentions. Diabetic Medicine 2000; 18: 835-841. 